\title{
Corela
}

Cognition, représentation, langage

11-2 | 2013

Vol. $11, \mathrm{n}^{\circ} 2$

\section{Cet obscur objet de désir : à propos de la construction want $G N$ et de ses gloses}

\section{Philippe Muller}

\section{OpenEdition}

\section{Journals}

Édition électronique

URL : http://journals.openedition.org/corela/2997

DOI : $10.4000 /$ corela.2997

ISSN : $1638-573 \mathrm{X}$

\section{Éditeur}

Cercle linguistique du Centre et de I'Ouest - CerLICO

\section{Référence électronique}

Philippe Muller, "Cet obscur objet de désir : à propos de la construction want GN et de ses gloses", Corela [En ligne], 11-2 | 2013, mis en ligne le 25 novembre 2013, consulté le 01 mai 2019. URL : http:// journals.openedition.org/corela/2997 ; DOI : 10.4000/corela.2997

Ce document a été généré automatiquement le 1 mai 2019.

\section{(c) (i) (2)(2)}

Corela - cognition, représentation, langage est mis à disposition selon les termes de la licence Creative Commons Attribution - Pas d'Utilisation Commerciale - Partage dans les Mêmes Conditions 4.0 International. 


\title{
Cet obscur objet de désir : à propos de la construction want GN et de ses gloses
}

\author{
Philippe Muller
}

\section{Rappels diachroniques: les grandes notions exprimables par l'énoncé en want}

1 L'évolution sémantique de want est connue et résumée par les lexicographes grâce à trois grands verbes quasi-synonymes chargés de fixer son évolution diachronique à travers un enchaînement: lack - require - desire. O. Polge $(2006,2007)$ à la suite de M. Krug (2000) montre très clairement que l'on " passe » du manque au désir, via la nécessité, à travers une «subjectivisation » car il y a introduction systématique d'un sujet animé humain $[\mathrm{AH}]$ là où want ne faisait apparaitre au départ qu'un objet manquant en position de sujet. C'est le cas en (1) où obedyence est l'argument unique de want :

(1) Where obedyence wanteth (sayth saynt Austyn) there is no goodness. (OED / 16 ème siècle.)

2 On peut en fait déjà considérer le manque comme un construit car on assiste à la mise en place d'une première opération de localisation ${ }^{1}$ LOC 1 par laquelle un objet $\mathrm{p}$ est repéré en absence par rapport à une situation de référence qui devient caractérisée par cette absence elle-même. (1) se traduit en effet par « là où il n'y a pas d'obéissance, il n'y a pas de bonté ». Alternativement, et dès le $16^{\text {ème }}$ siècle, la situation devient codable par there et rentre explicitement dans la prédication :

(2) For there wanted aboue sixe thousand souldiers of them which had been leuied.

(OED / $17^{\mathrm{ème}} \mathrm{s}$.)

3 L'on obtient alors un ordre repère-repéré. Le manque peut parallèlement être rapporté à un [AH] en position de premier argument (3) :

(3) We haue all sinned and want the glory whiche before God is allowed. (OED / 16 ème $s$.) 
4 L'ordre repère-repéré est maintenu par le placement de l'[AH] en position de sujet siège du manque. C'est cette dernière construction qui va s'imposer, ce qui va confirmer le statut de localisateur du sujet $\mathrm{S}$ par rapport à l'objet de manque p. On peut d'ailleurs parler d'une «réélaboration" de LOC1, à la manière de P. Cotte (1998) pour rendre compte du passage de (1) à (3).

5 L'objet $\mathrm{p}$ est en outre valué positivement puisque sa présence est attendue dans la situation de référence centrée sur le sujet, ce qui est assez clair en (4), extrait d'une recette de cuisine :

(4) If you want Peaches, you may use Juice of soure Apples. (OED / 17ème s.)

6 Le fait d'associer manque et permission modale montre que l'absence appréhendée du point de vue de $\mathrm{S}$, n'est pas indifférente à l'énonciateur mais fait l'objet d'une appréciation. Il y a bien sélection d'un objet manquant (peaches) utile pour la recette, que l'énonciateur juge donc nécessaire dans la situation de référence du point de vue de you, tout en autorisant (avec may) son remplacement par autre chose (the juice of soure apples). Si vous manquez des pêches nécessaires à la recette, vous pouvez utiliser du jus de pomme. Il envisage donc ici, en lien avec cette valuation, de pallier le manque de pêches.

7 M. Krug peut ainsi insister sur le fait que le désir émerge à partir du manque à travers la perception d'une nécessité qui naît d'une réanalyse pragmatique de l'énoncé en want (inspirée de ce que E. Traugott (1989) appelle "pragmatic strengthening »). C'est parce que le sujet d'énoncé ne dispose pas, dans la situation particulière dont il est le centre, du référent du second argument valué positivement que celui-ci lui est nécessaire et qu'il se trouve requalifié ${ }^{2}$ comme étant en position de désirer entrer en possession de cet objet de manque. On envisage alors avec want le dépassement nécessaire du manque, et cette nécessité est motivée par le désir supposé du référent du sujet dans la situation. Cela est concomitant de l'élargissement de la complémentation aux prédicats en to, ce qui devient très fréquent au $18^{\text {ème }}$ siècle. L'objet de manque $\mathrm{p}$ devient alors assimilable à une relation virtuelle ${ }^{3}$.

8 Nous pensons que la réinterprétation du manque au désir pour ce type de construction est possible au moins depuis le $16^{\mathrm{ème}}$ siècle :

(5) Like beggers wee liue and want to pay rent. (OED / 16ème s.)

9 En tant que pauvre, l'énonciateur ne peut pas payer le loyer qui lui est nécessaire mais on peut supposer que s'il le pouvait, il le ferait (ce qui est dit grâce au prédicat en to), le coénonciateur peut donc en déduire qu'il le veut ou qu'il le désire et réanalyse la prédication en conséquence.

Il faut retenir que le passage du manque au désir se fait d'abord à travers la mise en place avec want d'une transitivité de surface qui donne le second argument de want comme objet de manque. Emerge ensuite la possibilité de concevoir celui-ci comme une relation virtuelle introduite par to dont on peut dire qu'il pose syntaxiquement une altérité notionnelle sujet / prédicat (H. Adamczewski et C. Delmas (1998)). On peut dès lors utiliser want pour dire la palliation du manque dans l'extralinguistique car la relation est donnée comme validable et la construction nominale want GN devient elle-même comprise comme la possibilité d'envisager l'accès à ce qui fait l'objet du manque $\mathrm{p}$, ce qui le donne aussi comme objet de désir. Cette possibilité de l'assimilation d'une relation à $\mathrm{p}$ est ce qui constitue le fondement à la fois des hypothèses de réécritures que nous critiquerons et de notre propre proposition. C'est en effet la conception linguistique de l'accessibilité de p qui nous intéressera ici. 
11 Notons que c'est l'emploi archaïque dit « intransitif » que les dictionnaires rattachent au manque alors que want GN, construction de forme transitive, s'associe clairement à son dépassement. Want appelle normalement en synchronie un objet syntaxique, sauf cas d'ellipse (voir J. et F. Aarts (1995)). Le manque et rien que le manque n'est guère plus exprimable aujourd'hui sauf dans un emploi nominal (6) qui se traduit souvent par à défaut de..., ou dans un emploi quasi-adjectival (7) où le verbe reçoit-ing, est suivi de la préposition in, et peut commuter avec absent :

(6) For want of a better location, we ate our picnic lunch in the cemetery with superb views of the north coast. (BNC)

(7) [...] the antennae are often reduced to minute tubercles or are atrophied. They

are wanting in all Protura. (BNC)

12 La même notion est également évoquée lorsque le verbe se combine avec for et un GN qui se réduit à nothing ou anything, c'est-à-dire référentiellement à une non-entité comme en (8). L'ensemble prend alors le sens de « ne manquer de rien » :

(8) I have worked long and hard to ensure that my children want for nothing. (BNC)

On peut dire que ces constructions sont précisément des moyens de détransitiver want et ce faisant de le ramener vers son «sens premier ». A l'inverse, la mise en place d'une construction en apparence transitive s'accompagne, d'une manière ou d'une autre du dépassement du manque qui permettra d'exprimer le désir et la volonté, c'est-à-dire la palliation du manque.

\section{Point de vue adopté sur la synchronie}

14 Nous irons ici contre l'idée d'un «blanchiment sémantique » par lequel la notion de manque ne serait plus aujourd'hui exprimée par l'énoncé en want mais nous la prendrons comme le point de départ à partir duquel on peut construire le désir et la volonté. Ainsi, pour convaincant que soit l'enchaînement notionnel évoqué ci-dessus pour exprimer l'émergence du désir en diachronie, nous ne pensons pas que l'usage moderne de want nécessite que l'on pose "l'oubli» de ses acceptions antérieures. On peut d'ailleurs remarquer qu'en diachronie le désir n'a pas réellement remplacé le manque. Want au présent simple peut en effet continuer à exprimer le manque de façon stricte au moins jusqu'au $19^{\text {ème }}$ siècle comme le montre (9) qui servait à exprimer l'heure. Les deux aires sémantiques peuvent donc coexister au moins jusqu'à cette époque :

(9) It only wants five minutes to dinner. (OED / 19ème $\mathrm{s}$.)

Nous avons proposé ailleurs (P. Muller (2011)) que la notion de nécessité reste convoquée par ce verbe et doit être intégrée à son programme de sens car elle est l'un des sousproduits de l'opération LOC1 qui reste en jeu au niveau prédicatif et nous avons repris la notion d' " empathie » à S. Kuno (1987), C. Boisson (1987) et surtout P. Larreya (1989) pour éclairer le fonctionnement du type particulier de nécessité mis en jeu. Nous la pensons particulièrement évidente dans des exemples comme :

(10) I love Ben's look on his face; he wants a sip so badly! (Google)

(11) David Beckham Runs Through LA in His Underwear: Yes, You Want to Watch This!

(Google)

16 Nous chercherons ici à traiter du dépassement du manque en l'intégrant au programme de sens contemporain de want, en opposition aux théories de la désémantisation. Il s'agira de défendre l'idée que $\mathrm{p}$ reste d'abord un objet manquant, quelles que soient les notions - 
désir ou volonté - qui se construisent par ailleurs. C'est ainsi que (12) renvoie tout à la fois au manque, à la nécessité et au désir sans que ces notions ne s'excluent mutuellement :

(12) VLC Media Player Available for iPad, But We Still Want FLV Playback (Google)

Laissant de côté la nécessité, nous allons essayer de montrer comment le désir et la volonté s'envisagent par rapport au manque en travaillant sur la construction want GN qui a souvent été l'objet de réécritures systématiques.

\section{La construction want GN évoque sémantiquement à la fois le manque et sa palliation}

18 Want est souvent perçu par les locuteurs comme le complémentaire linguistique de have: il est reformulable par not (to) have. Le référent du sujet de ce prédicat veut ou désire parce qu'il n'a pas et nous avons montré à quel point les lexicographes exploitaient cette réécriture dans leurs gloses (P. Muller (2011: 28-31)). L'énoncé (13), qui illustre l'acception de type lack, montre que l'énonciateur associe les deux lexèmes pour les opposer à travers would rather qui marque l'expression d'une préférence distinguée entre deux termes qui doivent par ailleurs être sémantiquement comparables :

(13) A worthless old play-fellow of mine, whose company I would rather want than have. (OED / 1 gème $^{\text {s.) }}$

Or, de façon intéressante, l'échange (14) où want est utilisé dans son sens moderne de desire, témoigne de la même opposition :

(14) A : Don't make me hate you Syed.

$\mathrm{B}$ : Why would you hate me?

A : Cos I hate anything I want. That I can't have. (Eastenders)

Il illustre bien le fait que le désir est évoqué à partir du moment où l'on dépasse le simple constat du manque construit par LOC1 et que l'on envisage sa palliation, c'est-à-dire, d'une certaine façon, « une remédiation par l'avoir ».

Nous défendrons ici une hypothèse "surfaciste » en vertu de laquelle want GN suffit à évoquer ce dépassement. Ceci n'empêche pas qu'une glose par un second prédicat qui explicite la palliation du manque reste reconstructible. C'est à cette relation, à son statut et aux différentes manières dont ont peut la concevoir que nous nous intéresserons particulièrement. Remarquons en effet, à partir de (13) et (14), que cette relation est codable explicitement plus loin dans le cotexte, ici par have.

De façon intéressante, I. Birks (2002) montre que chez l'enfant le verbe have apparaît après want. Tout se passe comme si le premier émergeait à partir de ce dernier. Il souligne ainsi qu'en réponse à une question en want posée par un adulte, l'enfant produit d'abord un énoncé où il substitue un autre verbe qui implique le passage à l'action susceptible de combler le manque (il répond ainsi au « you want a bath ? de l'adulte par «I take a bath »). C'est que want est compris comme l'expression du désir de ce qui n'est pas encore actualisé pour son sujet mais peut le devenir. Le fait que la question en want soit prononcée par un adulte, c'est-à-dire quelqu'un susceptible d'exercer un pouvoir sur l'enfant et sur son monde, pousse celui-ci à analyser le verbe comme ce qui permet d'exprimer ce qui annonce une dévirtualisation. Autrement dit, want serait compris initialement par l'enfant comme ce qui précède la palliation du manque que le désir implique ${ }^{4}$. Birks montre que le verbe have, à partir du moment où il apparaitt, est analysé 
comme le dépassement du désir. C'est pour cela qu'il émergerait cognitivement à partir de want, dont il serait en quelque sorte « la négation métalinguistique » $(2002: 296)$.

Nous faisons alors l'hypothèse que l'énoncé en want permet d'envisager notionnellement à la fois un manque et son dépassement nécessaire par une seconde relation que le second argument doit, d'une manière ou d'une autre, permettre d'évoquer et nous verrons que certains ont justement cherché à systématiser ce dépassement au niveau syntaxique en intégrant have - ou d'autres verbes - au fonctionnement prédicatif de want.

\section{Le second argument $p$ comme objet de manque globalisé}

Afin d'étudier le dépassement du manque, nous partirons de l'idée simple que quelle que soit la complémentation utilisée avec want, le second argument est assimilé sémantiquement à un objet comme le montre le fait qu'il corresponde toujours à une question en what. Nous ne prendrons qu'un exemple, révélateur à cet égard, car le second argument en est extrêmement réduit. Il relève de ce que l'on peut analyser comme une préposition ou une particule ${ }^{5}$ :

(15) Phil, your boy wants in. (Eastenders)

(16) A : What does your boy want ? B : He wants in.

(17) A : * Where does your boy want ? B : He wants in.

Autrement dit, le complément a bien une sémantique d'objet qui peut d'ailleurs renvoyer à une seconde relation bien plus explicite :

(18) A : What does he want ? B : To be let in.

Cette assimilation d'une relation ou d'un élément relationnel à un objet semble bien être imputable à want lui-même qui, à travers LOC1, donne le référent de $\mathrm{p}$ comme un objet de manque. C'est ainsi que in, en isolement de want ne semble plus faire sens :

(19) A : What does he want ? B :? ? In.

Autrement dit, ce verbe a la propriété de globaliser son second argument $\mathrm{p}$ et de le donner comme non-immédiatement disponible ( =manquant) pour son sujet $\mathrm{S}$.

Par ailleurs, on peut rappeler que la syntaxe du second argument n'est pas une syntaxe transitive, dans la mesure où la passivation est difficile (20), sauf dans les énoncés de type " avis de recherche » (21), ce qui tend à montrer que le GN, dans la construction want GN, n'est pas directement régi par want d'un point de vue syntaxique :

(20)? ?An ipad is wanted 6 .

(21) He was unwanted as a child and now he's wanted in five states. (Time corpus)

Or, cela peut s'interpréter sémantiquement, pour la complémentation nominale qui nous intéresse ici, comme le fait que le référent du GN renvoie souvent avec want à quelque chose de plus qu'un objet, ou une chose, c'est-à-dire une "entité de premier ordre » (J. Lyons (1995)). Nous disons en tout cas que want GN permet de construire un manque à travers LOC1, mais que cette notion est en réalité aujourd'hui systématiquement dépassée dans la mesure où la construction en évoque déjà la palliation. Nous examinerons ici deux idées-forces.

La première est que want rend le référent du GN directement support d'une visée et le donne en fait comme objet de désir, c'est-à-dire qu'il devient conceptualisé comme susceptible d'intégrer la sphère du sujet d'énoncé simplement par «projection dans 
l'avenir » (M.-L. Groussier et C. Rivière (1996)), car ce prédicat marque en lui-même « un objectif à atteindre et un hiatus (une distance à combler)» (A. Culioli (1990: 133)) :

(22) Roberto Orci Says Paramount Wants Star Trek XIII in 2016.

"They are firm about having a movie out by the 50th anniversary [in 2016] and we

are all trying to see if we can all make it work together". (Google)

Nous reviendrons sur ce point à la section 16 après avoir examiné la possibilité d'une seconde relation sous-jacente, systématiquement présente, qui pourrait expliquer les paraphrases rencontrées en (13) et (14) et dont certains ont fait le fondement même de l'accessibilité du référent du GN. Nous nommons cette relation LOC2, afin de rendre compte de l'altérité cognitive qu'elle suppose ${ }^{7}$. Les gloses en have peuvent alors se comprendre justement comme une des matérialisations possibles de cette accessibilité. L'échange (23) fait ainsi apparaître une explicitation spontanée de LOC2 par des locuteurs qui ont bien compris que ce qui est en jeu avec want GN, c'est l'accession du sujet d'énoncé à l'objet de désir :

(23) A : I want that as well.

$\mathrm{B}$ : You're not having that ! (BNC)

La déconstruction partielle de cette seconde hypothèse nous ramènera alors naturellement à la première et notre proposition de traiter le second argument de want comme un domaine notionnel est à comprendre comme une façon de tenter la conciliation de ces deux idées ${ }^{8}$.

Précisons notre conception des notions de désir et de volonté.

\section{Manque, désir et volonté comme notions connexes représentables en domaine notionnel}

Le Vocabulaire technique et critique de la philosophie de Lalande (2006), permet de poser une distinction désir / volonté :

«Désir: Tendance spontanée et consciente vers une fin connue ou imaginée. Le désir repose donc sur la tendance dont il est un cas particulier et plus complexe. Il s'oppose d'autre part à la volonté (ou à la volition) en ce que celle-ci suppose de plus : $1^{\circ}$ la coordination au moins momentanée des tendances; $2^{\circ} l^{\prime}$ 'opposition $d u$ sujet et de l'objet ; $3^{\circ}$ la conscience de sa propre efficacité $; 4^{\circ}$ la pensée des moyens par lesquels se réalisera la fin voulue. »

Il en ressort que le désir est définissable positivement comme un cas particulier de tendance'. ${ }^{9}$ Il est alors à rattacher, dans le domaine linguistique, à l'opération de visée et peut se laisser appréhender comme le fait d'envisager l'entrée potentielle d'un objet de manque nécessaire dans l'univers du sujet d'énoncé.

Par ailleurs on peut définir le désir négativement, grosso-modo comme une volonté sousdéterminée. Cette deuxième notion implique en effet une détermination plus grande en ce qu'elle porte, non-seulement, sur un objet plus clairement circonscrit d'un point de vue référentiel, mais également sur les moyens et les stratégies à mettre en œuvre dans l'extralinguistique pour rentrer en possession de cet objet.

Dans le passage suivant, le narrateur joue sur la dimension inconsciente du désir du personnage. On notera le contexte largement négatif et l'évocation d'un décor instable à travers des formes faiblement déterminées d'un point de vue référentiel (no one, somewhere) : 
(24) Arcadia Darell, dressed in borrowed clothes, standing on a borrowed planet in a borrowed situation of what seemed even to be a borrowed life, wanted earnestly the safety of the womb. She didn't know that was what she wanted. She only knew that the very openness of the open world was a great danger. She wanted a closed spot somewhere - somewhere far - somewhere in an unexplored nook of the universe - where no one would ever look. (I. Asimov, Second Foundation) volonté contienne notionnellement un désir et nous proposons, pour expliquer les liens qui relient want $G N$ et want to $V G N$, d'utiliser le modèle topologique culiolien de structuration d'un domaine notionnel ${ }^{13}$ :

\section{Volonté Désir}

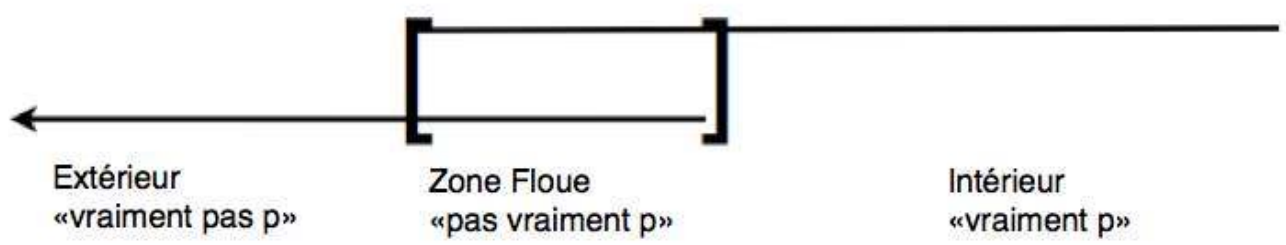

Ce schéma, qui est à comprendre comme la représentation des notions exprimables du point de vue du sujet $\mathrm{S} 1$, permet de rendre compte du fait qu'avec want, le second argument $p$ est conceptualisé globalement comme un objet de manque qui peut - à partir du moment où l'on fait émerger des discontinuités cognitives pensées en rapport avec ce qui en constitue « vraiment l'objet » - exprimer des notions connexes qui ne s'enchainent pas de façon étanche, comme le laisse entendre l'analyse diachronique, mais s'activent 
mutuellement. Nous allons clarifier notre point de vue à partir d'une lecture critique des hypothèses qui proposent une réécriture systématique de want $G N$ au moyen d'un second prédicat.

\section{L'hypothèse du dépassement syntaxique systématique de want $G N$}

Une partie de la tradition linguistique américaine a tenté de réécrire systématiquement want $G N$ au moyen d'un second prédicat verbal, ce que nous comprenons ici comme une façon de matérialiser une altérité cognitive dans la complémentation en relation avec la notion évoquée par le référent du GN que le prédicat want, donne d'abord comme objet de manque. Il s'agit en effet de considérer qu'une seconde relation imbriquée est toujours implicitement recouvrable à partir du référent $d u$ GN qui permet d'envisager linguistiquement la relation qui le donne comme accessible dans l'extralinguistique. Nous dirons que la première série d'hypothèses que nous abordons ici, qui cherche à systématiquement gloser want GN par want to $V$ GN repose sur un non-dit en ce qui concerne leur proximité sémantique : l'idée que l'on peut expliquer sémantiquement ce qu'est un objet de désir en explicitant une volonté d'en faire quelque chose ${ }^{14}$. La section 7 montrera, par exemple, que l'on peut expliquer ce qu'est un désir de cigarette à travers une volonté de la fumer.

\section{Critique de l'effacement systématique d'un prédicat enchâssé en to $V$}

J. Pustejovsky (1995) propose qu'un verbe comme want possède un complément de nature «foncièrement événementielle ». Le référent $d u$ GN qui renvoie à l'objet à la fois manquant et désiré va contraindre, par " coercition de type ", la construction du procès qui permet d'en envisager l'existence du point de vue linguistique. Reste à savoir si celuici est envisagé de façon sémantique ou syntaxique. J. Fodor et E. Lepore (1998) optent pour la deuxième hypothèse. Ils interprètent les travaux de Pustejovsky comme signifiant que (24) se laisse « parfaitement » gloser par (25):

(24) I want a cigarette.

(25) I want to smoke a cigarette.

De la même manière, (26) serait « synonyme » de (27) :

(26) I want a beer.

(27) I want to drink a beer.

Un nom comme book supposerait l'effacement du procès interpolé read, sandwich celui de eat, etc... Puisque le référent du GN est vu comme le dernier point du procès qui l'amène à l'existence, on peut penser que le procès récupérable est nécessairement de nature télique ${ }^{15}$ et implique un sujet co-référent à celui de son verbe recteur, want, ce que seul $\mathrm{R}$. Dixon (1991 : 185) prenait la peine de préciser. Dans cette hypothèse, le référent du GN permet d'évoquer l'aboutissement du procès supposé effacé. Pour être plus précis, le procès en question semble même devoir être de type "accomplissement" dans la classification de Vendler (1967) ${ }^{16}$. Cette interprétation de ce que propose Pustejovsky qui implique l'interpolation systématique d'une seconde relation - a ensuite été discutée par S. Wechsler (2005) et F. Schwarz (2006) qui objectent que lorsqu'un quantificateur est 
présent avec want $G N$, on pourrait penser qu'il se distribue systématiquement sur la deuxième relation. Or, ils s'opposent vigoureusement à cette prédiction car (28) n'est pas synonyme de (29) :

(28) I want a beer in two minutes.

(29) I want to drink a beer in two minutes.

(28) signifie plutôt le désir d'être mis en présence d'une bière dans deux minutes que le fait de vraiment vouloir boire sa bière en deux minutes, comme (29). Ainsi, la quantification évoquée par in two minutes en (28) semble porter sur le premier procès, celui évoqué par want, plutôt que sur un éventuel second procès imbriqué et effacé. D'une part want $G N$ semble bien sémantiquement permettre de faire référence à la palliation du manque mais, d'autre part, les quantificateurs ne portent pas systématiquement sur un second procès interpolé dans la syntaxe. Nous disons alors que want GN n'est pas toujours le résultat d'un effacement.

Notre propre hypothèse repose sur les deux points suivants :

1. Le second argument de want est globalisé comme un objet de manque mais celui-ci est susceptible d'intégrer notionnellement des discontinuités plus ou moins explicitables et pensées à partir de ce qui constitue ce qui est "vraiment p », l'objet du manque au sens strict, qui organise l'intérieur du domaine notionnel.

2. La construction want GN évoque un dépassement du manque déjà acquis notionnellement qui donne le référent de son objet comme objet de désir dans la mesure où il peut faire directement l'objet d'une visée, indépendamment d'une seconde relation LOC2.

51 Le fait d'envisager le second argument comme un domaine notionnel nous permet de concilier ces deux points en n'obligeant pas à concevoir le dépassement du manque comme nécessairement construit syntaxiquement, ce qui va contre l'idée de l'effacement, mais ce qui n'écarte pas non plus la possibilité de la reconstruction d'un second prédicat.

\section{La possibilité d'une reconstruction}

Le fait que la construction want GN ne soit pas systématiquement la "réduction » de want to $V$ GN ne signifie pas qu'elle ne puisse parfois l'être ou être interprétée comme telle. Si (28) et (29) ne militent pas en faveur de l'effacement systématique, en (30) la présence d'un prédicat télique présent dans le cotexte avant (contact) semble être ce qui autorise qu'on utilise ensuite want $\mathrm{GN}$ pour évoquer directement l'accession au référent du GN, Starfleet Command, comme si l'on « court-circuitait » le second procès :

(30) Tonais whirled toward Milan. "You! Contact Starfleet Command!" Shira followed the director. "Starfleet Command? Don't you mean the nearest Federation representative, sir? Ambassador Li -- " "No, I want Starfleet Command. They're the only ones who can handle this situation now." (David Mack, Star Trek CCE : Creative Couplings)

Dans cette interprétation on peut dire qu'une volonté de communiquer est implicitée, ce qui permet de ne l'évoquer ensuite que par le biais du référent du GN désignant le groupe de personnes avec qui l'on désire parler (Starfleet Command). Pour autant, dans un contexte lié aux télécommunications comme celui-ci, il n'est même pas nécessaire de mentionner un procès comme contact ou be put in touch with qui est présupposé par la situation. Autrement dit, want $G N$ peut être le point de départ de la reconstruction d'un 
second procès en to $V$ qui permet d'en expliciter le statut d'objet de désir en indiquant par quel procès télique il devient accessible.

Le modèle topologique permet de revenir aux exemples de la section précédente et de dire qu'avec want $G N$ on se situe a priori en deçà de la problématique de la frontière au sein du second argument $\mathrm{p}$ globalisé notionnellement comme objet de manque. Cette construction permet d'envisager une palliation du manque à travers la prise en compte d'une altérité qui n'est évoquée que de manière embryonnaire, dans la mesure où l'on peut penser qu'il y a indistinction notionnelle entre l'objet du manque, un éventuel procès explicitable à travers lui et une seconde subjectivité S2 coréférente avec S1 qui pourrait lui servir de relais dans le virtuel. Cela n'empêche pas la reconstruction éventuelle d'un second procès en to $V$ de type accomplissement qui permet d'expliciter l'accessibilité au référent du GN qui le donne en même temps comme l'aboutissement d'une "volonté de faire ». C'est ainsi qu'expliquer le statut d'objet de désir du référent du GN peut passer par l'explicitation de ce que l'on peut vouloir faire avec.

\section{Want GN et le procès reconstruit sont repérés en altérité faible}

55 Want GN et want to $V G N$ sont donc des constructions distinctes et, avec la première, on reste en deçà de la question processuelle, même si on peut les unir par un lien paraphrastique dont nous cherchons à expliquer la possibilité sans toutefois en défendre la systématicité. La prise en compte de ce que nous avons appelé "altérité cognitive » construite à partir du référent $\mathrm{du} \mathrm{GN}$, qui fonde le dépassement du manque que constitue le désir, ne nécessite donc pas l'effacement d'une relation sous-jacente comme le confirme (31), l'objet de manque pouvant être visé directement :

(31) I want a quick tattoo.

56 Pour autant cet énoncé peut signifier plus. Veut-il dire en effet qu'un second procès qui amènerait le passage à l'existence du tatouage est vite réalisé ou bien l'adjectif quick porte-t-il sur le rendu du tatouage, l'état résultant du procès? Il n'est pas possible de trancher hors contexte, comme le montrent (32) et (33) qui mettent respectivement en valeur les deux interprétations :

(31) These potential customers wanted to do a quick tattoo, but Kelvin answered: "If you want the tattoo to be good, it needs time." (Google)

(32) This is a quick tattoo I drew on my way home one day, that took me about 5 minutes. It's a drawing of H.P. Lovecraft's monster Cthulhu. [...] All Lovecraft fans tell me whatcha think. (Google)

On peut ainsi éventuellement viser un second procès implicite à travers le GN qui en évoque l'aboutissement. Le modèle topologique permet de dire qu'il y a, dans l'argument p globalisé comme objet de manque, une partie qui correspondrait à ce qui est « vraiment $\mathrm{p}$ » et une partie qui lui est assimilable et participe à sa détermination. Ainsi la glose en want to $V$ GN dont nous avons précisé les conditions ci-dessus, peut servir à préciser le sens de want $G N$ car la reconstruction du second procès ajoute à la détermination du référent du GN. On pourrait parler de "détermination processuelle» de celui-ci. Elle indique comment - à travers quelle relation - l'énonciateur envisage son passage à l'existence, son accessibilité en tant qu'objet de désir. Le modèle topologique illustre bien le fait que le référent du $\mathrm{GN}$ se situe dans le prolongement du procès reconstruit et vice versa. 

whisky, auquel on peut remédier (car want permet de viser le référent du GN), ce qui permet de construire notionnellement un désir de whisky pour le sujet d'énoncé. Ce désir de whisky peut par ailleurs s'intégrer lui-même à une volonté de boire un whisky exprimable par «I want to drink a whisky». On introduit alors un relais subjectif implicite S2, correspondant ici au PRO des générativistes, qui constitue l'objet - ou le support - de la volonté de I et qui est alors extériorisé, distingué de ce qui est visé grâce à to qui matérialise une frontière dans $\mathrm{p}$. Ce qui est visé, n'est plus simplement un whisky, mais le procès désigné par drink $a$ whisky ce qui donne le prédicat reconstruit en objet de désir complexe ${ }^{17}$ pour le sujet de want. Le whisky et le fait de le boire deviennent quasiment indistinguables tant ils sont assimilés l'un à l'autre, ce qui explique les relations paraphrastiques entre les deux constructions, le référent du GN étant, dans ce cas particulier, l'aboutissement notionnel à la fois de want GN et de want to V GN.

Pour autant, comme le GN est aussi le point de départ de la reconstruction proposée, on gagnerait à envisager la relation entre le second procès postulé et le référent du GN comme bidirectionnelle, ce que nous proposons nous-même. Le référent du GN n'est alors plus seulement un " point d'arrivée », mais tout autant un « point de départ » notionnel.

Il faut d'ailleurs noter qu'en réalité Pustejovsky ne défend pas vraiment l'interpolation, comme le pensent Fodor et Lepore. Pour lui le second procès est intégré à ce qu'il appelle la «structure de qualia » de want qui a la particularité d'évoquer un événement dans sa complémentation, d'en permettre la construction par « co-composition». Et il proposait de gloser (26) par (34) plutôt que par (27) :

(34) I want a beer (to drink).

61 En tout cas, la relation de palliation, qui n'a évidemment pas de statut syntaxique dans want $\mathrm{GN}$, n'est au départ pas clairement distinguée cognitivement de l'objet de manque. Elle n'est explicitée qu'à partir du moment où cet objet de désir est distingué de $\mathrm{S} 2$, objet de la volonté de S1, à travers le second prédicat en to, ce qui suppose la prise en compte d'une altérité forte et marquée au sein du second argument $\mathrm{p}$ globalisé en objet de manque.

\section{Le procès reconstruit est un représentant d'une classe de procès}

En réalité, c'est J. McCawley (1974) qui a le plus clairement formulé l'hypothèse de l' interpolation systématique d'un verbe dit « léger » effacé. Elle consistait à dire que want GN est le résultat en surface de l'effacement d'un have initialement présent en structure profonde. Il s'agit en fait d'une idée que l'on peut au moins faire remonter à W.V.O. Quine (1960 : 155) pour qui want est sémantiquement le « raccourci » de wish to have (il parle de "shortcut verb »). McCawley avait étendu cette idée à la syntaxe et dans cette optique (26) se laisse plutôt gloser par (35). Là encore, il s'agissait pour lui d'énoncés « synonymes » :

(35) I want to have a beer.

De la même manière, (36) et (37) seraient équivalents :

(36) I want a cigarette.

(37) I want to have a cigarette.

Cette hypothèse, qui a aussi été défendue par J. Ross (1976), paraît à la fois plus souple et plus élégante que la précédente. En effet, la construction have a $N$ a souvent un sens dit 
"dynamique » et dans cette analyse, have est à concevoir comme un hyperonyme renvoyant à ce que nous dirons être une classe de procès téliques de type «accomplissement» dans la classification de Vendler et qui signifient plus ou moins consume. On voit que le référent $\mathrm{du} \mathrm{GN}$ joue un rôle primordial dans la construction de ce qui est un événement secondaire par rapport à want alors codable par have. Ceci a l'avantage d'éviter la multiplication des prédicats effacés (have a beer, se substituant avantageusement à drink a beer, have a sandwich à eat a sandwich, etc...). C'est sur ce terrain qui associe syntaxe profonde et interprétation sémantique que se placent Fodor et Lepore, dans une approche que l'on peut qualifier de «polysémie systématique ». Il faut pourtant noter que McCawley avait assis son hypothèse de l'interpolation sur des bases syntaxiques, à partir de la question de la portée des déterminations dont Schwarz (Op. cit.) rediscutera beaucoup plus tard, comme nous l'avons $\mathrm{vu}^{18}$. Cette hypothèse retombe donc sous le coup de la critique adressée en 7 et par laquelle nous avons conclu que want GN et want to $V G N$ renvoyaient à des conceptions différentes du dépassement du manque et sont en fait des constructions distinctes.

On voit en tout cas bien ce qui distingue la construction want to $V$ reconstruite à partir de want GN de son usage « en première intention » qui, il faut le noter, n'a pas de statut clair dans les hypothèses que nous critiquons. Dans le premier cas la construction est utilisée pour évoquer une possibilité parmi d'autres, alors que dans le second elle est précisément choisie pour sélectionner un prédicat unique qui n'est d'ailleurs pas forcément télique (38) et dont le sujet n'est pas nécessairement co-référent avec celui de want (39) :

(38) I want (PRO) to see you tonight.

(39) He wants her to be open minded.

\section{Problématique de la frontière et distinction d'un objet de volonté pour S1}

Dans notre analyse, le procès reconstruit appartient à la zone floue du domaine notionnel. Il explicite l'altérité faible que l'on peut concevoir à partir du centre du domaine que constitue «ce qu'est vraiment l'objet de manque» et dont il est difficilement distinguable. Ce second procès, qui est un possible parmi un ensemble de possibles, reste implicite et n'est pas fortement distingué de ce qu'est " vraiment p ", à moins que le désir ne soit paraphrasé au moyen d'une volonté de faire quelque chose de l'objet de manque qu'il suppose. Dans ce cas, le procès est explicité syntaxiquement et émerge à partir de ce dernier, «donnant à voir» son statut d'objet de désir. Il est alors séparé de ce qui constitue l'objet de la volonté de $\mathrm{S} 1$, le sujet $\mathrm{S} 2$, par une frontière matérialisant une altérité notionnelle forte avec cette deuxième subjectivité qui est le point de départ du procès qu'il code et qui permet de penser l'accession à ce qui est « vraiment $p$ » dans le second argument.

Dans «I want PRO to smoke a cigarette », PRO - correspondant à S2 dans S1 want S2 to V GN est la cible privilégiée de la volonté de S1. A l'intérieur du second argument $\mathrm{p}$, il est pensé à travers une altérité forte, marquée par to qui matérialise une frontière ou un «nexus " relationnel marqué (O. Jespersen (1992)). Cette frontière le distingue, d'une part, de ce qui est « vraiment l'objet de manque » dans son univers, la cigarette, qui aurait pu faire plus directement l'objet d'une visée par ailleurs comme avec "I want a cigarette », et d'autre part du prédicat verbal smoke. On voit alors qu'il ne s'agit pas simplement pour S1 d'exprimer un désir de cigarette mais une volonté de fumer une cigarette. Smoke permet 
de donner à voir le fait que la cigarette n'est pas qu'un objet de manque mais aussi un objet de désir distingué de S2 qui lui est extérieur. Pour autant, S2 n'est "pas complétement étranger » à cet objet de désir car il est donné par to en rupture par rapport à lui. Il lui est donc partiellement assimilable, en tant que partie intégrante de p qui est lui-même globalement conceptualisé comme un objet de manque. On voit donc bien comment la problématique de la frontière éclaire la sémantique de $\mathrm{p}$ car elle est à relier à l'émergence de la distinction sujet-relation-objet qui offre un support sur lequel la volonté de S1 peut s'exercer.

Par contraste, les énoncés en want qui contiennent un complément de type proposition réduite ("small clause») mettent en jeu un nexus relationnel non-marqué et ne distinguent pas entre support de la volonté et objet de désir. Ces énoncés semblent souvent avoir pour fonction d'impliciter la volonté de S1 à la faveur de son désir :

(40) "Frankly, I don't care," the girl said. "It's your problem, not mine. I just want you to get out of here. Right now. This is my place, and I want you out. (P. Auster, City of Glass.)

Cette construction est donc un moyen d'éviter volontairement la question processuelle tout en maintenant éventuellement en surface une altérité explicite sujet / élément prédicatif dans le second argument. Le second procès reste un non-dit et c'est pourquoi (41) est une dénégation:

(41) She says she did want him out of the house, but she didn't want to kill him as a court was going to evict him anyway. (BNC)

"She wanted him out of the house " ne spécifie effectivement aucun procès, simplement un état résultant pensé à partir d'une relation du type him / (be) out of the house. Le sujet she nie l'inférence selon laquelle le tuer aurait été le meilleur moyen pour elle d'atteindre cet état désiré. La prise en compte d'un second repère subjectif dans le second argument, sans prise en compte du nexus, est ce qui crée l'ambiguité désir / volonté qui risque d'incriminer l'énonciatrice devant le tribunal. De même, en (42) tiré d'un article en ligne de $\mathrm{CNN}$, une mère envisage d'abord simplement la mort du référent de him, le tueur de son enfant («I want him dead»). Le fait de mourir ou de ne pas mourir, se donne comme étant envisagé en lien avec cette instance subjective dont il est peu différencié. Le cotexte met pourtant rapidement en valeur le fait que ce qui est souhaité, c'est l'intervention d'un tiers à travers un prédicat explicite (be put to death) pour actualiser l'état résultant, mais cela était au départ un non-dit :

(42) "He couldn't be dead fast enough for me. I want him dead. I want him dead now ." Asked if it mattered whether Smith was put to death or spent the rest of his life behind bars, Schorpen firmly stated, "My daughter's not breathing. She'll never breathe again. I can never hold her again. I've got to wait for appeals before, you know, he dies? It matters to me." (Google)

71 La volonté sous-jacente qui serait rendue visible par une "structure manipulative» comme " I want somebody to put him to death » qui matérialiserait la relation qui mène à l'état résultant souhaité n'est pas assumée par l'énonciatrice.

72 Notre hypothèse sur la structuration du second argument de want est ce qui permet de faire émerger la problématique de la frontière dans l'interprétation de l'énoncé où ce verbe apparait. Elle permet de prédire de la même manière que la construction want GN Ving, qui intègre un nexus relationnel non-marqué est à relier à l'expression du désir :

(43) I want him waiting by this corner. (BNC) 
Ainsi, à la forme négative ( I I don't want him waiting by this corner »), la négation ne porte pas sur le nexus lui-même. Ce qu'exprime l'énonciateur est moins une volonté niée, le rejet d'une action que S2 pourrait commettre, comme cela serait le cas pour «I don't want him to wait by this corner ", qu'un non-désir de le placer dans une position défavorable. Notons que cette position peut être défavorable pour S1 ou pour S2:

(44) I don't want him sneaking around, searching through my drawers and cupboards and trying to find my diaries. (Google)

(45) I don't want him waiting on me ! I've told him that repeatedly. He can't start to get over me if he can't even grasp that we're over ! (Google)

\section{L'hypothèse de la « préposition abstraite »}

Après avoir critiqué les théories de l'effacement, nous nous tournons vers une autre hypothèse qui va nous permettre de préciser notre analyse de la sémantique de want GN. H. Harley (2004) a fait un pas supplémentaire vers l' « abstraction » de la seconde relation postulée pour want GN, en proposant que ce qui est effacé n'est pas un verbe have mais une "préposition abstraite» appelée $\mathrm{P}_{\text {have }}$. Celle-ci est potentiellement contenue dans d'autres verbes que have, par exemple get. Elle part ainsi d'un exemple comme :

(45) I want a kiss / a compliment.

75 (45) ne signifie pas «I want to have a kiss / a compliment » mais se laisse plutôt gloser par (46) :

(46) I want to get a kiss / a compliment.

76 Il s'agit sémantiquement de les recevoir ${ }^{19}$. Harley cherche alors ce qu'il y a de commun aux verbes have et get et propose de les réécrire à l'aide de la "préposition abstraite » $\mathrm{P}_{\text {have }}$. Ainsi, have a une dimension "stative ", et pourrait donc se réécrire par $\mathrm{BE}+\mathrm{P}_{\text {have }}$ alors que get a une dimension "dynamique », et se réécrirait BECOME $+P_{\text {have }}$. L'hypothèse du relateur abstrait est sa façon de proposer une relation de palliation du manque qui lève l'objection syntaxique de la distribution systématique des quantifieurs. Il s'agit d'une hypothèse "plus sémantique $»^{20}$. Ce qui est proposé est en fait l'idée que want GN correspond toujours à une proposition réduite ou «small clause $»^{21}$ organisée autour de la préposition abstraite. Cette dernière aurait des propriétés différentes du verbe have mais permettrait de maintenir sémantiquement l'idée d'une entrée virtuelle de l'objet dans le domaine du sujet grammatical. Nous comprenons alors la relation posée comme une LOC2 modulable en [+/- dynamique] mais à laquelle on n'accorde pas de réalité syntaxique. Pour intéressante qu'elle soit, l'hypothèse de la préposition abstraite nous semble aussi « unidirectionnelle » que celle de l'effacement, le référent du GN étant envisagé comme l'aboutissement de la seconde relation alors qu'il constitue également son "point de départ » éventuel, étant indispensable à l'affirmation d'un désir particulier, want n'étant assertable qu'à partir d'un objet de manque.

\section{Le référent du $\mathrm{GN}$ comme centre du manque et du désir}

Dans notre modèle topologique, un objet de manque est le prérequis de la détermination d'un désir ou d'une volonté, le point de départ de son affirmation par want, ce qui le constitue comme le centre du domaine notionnel envisagé à partir de son second 
argument. Cela s'explique par le fait que want, tout comme need, est classé parmi les "prédicats transitifs intensionnels", lesquels se définissent par deux grandes propriétés (voir, par exemple F. Schwarz (2006) et S. Harves (2008)) :

78 1. Une substitution extensionnelle de l'objet du verbe change la valeur de vérité de la phrase. Ainsi, «I want a beer », n'a pas la même valeur de vérité que "I want a lager ", même si lager est une sous-classe de beer. Ce phénomène a trait à ce que l'on peut appeler « individuation ». Le désir ou la volonté que want permet d'évoquer ne peut être comblé que par un objet ou groupe d'objets que ce verbe construit comme distingués d'autres objets ou groupes d'objets à travers la mise en relation particulière qu'il permet. L'objet de want est donc individué, car cet objet est vu comme susceptible de satisfaire un désir particulier. Il fait en retour partie de la « définition » de ce désir particulier.

79 2. Les prédicats transitifs intensionnels ne nécessitent pas l'existence de l'objet dans la situation pour former des phrases vraies (propriété souvent appelée «lack of existential import »). Dans « He wants a unicorn, flying candy, and a rainbow for Christmas» (Google), les référents des GN en question peuvent n'être pas présents dans le contexte situationnel sans que la vérité de l'énoncé soit remise en cause, ce qui est à associer à l'opération de visée sous-jacente.

Le modèle topologique et la notion de repérage empruntés à A. Culioli permettent de comprendre pourquoi cet objet de manque est non seulement le point de départ mais aussi le point d'arrivée de la relation reconstruite.

\section{Le référent du $\mathrm{GN}$ et le prédicat reconstruit entrent en relation de repérage}

81 Le modèle topologique que nous utilisons pour expliquer les relations paraphrastiques want $G N$ / want to $V G N$ est ce qui nous permet de dire que le prédicat verbal reconstruit constitue une extension du GN dans la mesure où il forme avec lui une notion complexe. Nous souhaitons montrer qu'ils entretiennent une relation de repérage bidirectionnelle et partirons de l'analyse de (47) qui permet de raisonner en dissociant le référent du GN de la seconde relation reconstructible:

(47) The food was inedible. I wanted chicken and the waiter insisted their crispy beef was the best. It was deep fried beef, jerky and overly sweet. (Google)

Dans "I wanted chicken", un prédicat est reconstructible, eat, à partir de chicken, GN second argument de want au prétérit correspondant notionnellement à un objet donné comme absent pour le référent du sujet, c'est-à-dire un objet de manque. Le fait que celuici soit second argument de want le donne en réalité déjà comme visé, ce qui en fait un objet de désir. Want permet d'ébaucher le dépassement du manque et de construire un domaine notionnel autour de lui.

Reconstruire le prédicat eat permettrait à la fois de déterminer davantage l'objet de manque / désir et d'envisager explicitement l'accès à ce qui constitue « vraiment $p$ » dans le domaine, à travers l'expression d'une volonté. Or, le prédicat eat, ne saurait être sélectionné ici à partir de la nourriture que l'énonciateur a effectivement reçue (crispy beef), comme le marque l'adjectif inedible. Avec want $\mathrm{GN}$, il doit y avoir adéquation entre le second prédicat reconstruit et ce qui constitue "vraiment $\mathrm{p}$ ». La nature qualitative de l'objet manquant est ce qui permet d'en juger. C'est pourquoi en (47), la réalité de l'objet 
effectivement reçu (crispy beef) ne permet pas vraiment de sélectionner le verbe eat, comme le dit l'énonciateur. On pourrait logiquement asserter (48) à partir de (47) :

(48) I didn't really want crispy beef. I wanted something else.

Si (47) montre que le GN et le prédicat reconstructible entrent dans une relation de repérage, (49) révèle que celle-ci fonctionne dans les deux sens :

(49) I thought I wanted a Big Mac the other day, so I made the husband go get me one. I ate like $1 / 4$ of it and it wasn't what I wanted at all. [...] I like BK better for fast

food. I think what I really wanted all along was an Italian sandwich. (Google)

Dans l'exemple (49), le Big Mac, référent du GN second argument de want est donné plus loin comme faisant l'objet d'un processus de consommation à travers l'assertion de la relation I / eat au prétérit. Celle-ci peut se comprendre comme la matérialisation contextuelle de la relation qui permet de pallier le manque au sens où elle permet indirectement d'accéder au Big Mac, conceptualisé comme ce qui constitue vraiment l'objet du manque. L'énonciatrice se rend compte ensuite que ce qui constituait référentiellement « vraiment $\mathrm{p}$ » était en fait autre chose, an Italian sandwich. Elle change sa façon d'envisager qualitativement ce qu'elle désir atteindre. Son « discernement » de l'objet de manque est différent. Ce que dit l'énoncé, c'est que le prédicat reconstructible sélectionné, eat (resté implicite dans la prédication en want), n'aurait pas dû permettre de déterminer le Big Mac comme objet de désir. Ce n'est pas ce prédicat qui est remis en cause ici, car il fait partie des présupposés de la situation, mais son objet final, son telos.

(47) et (49) montrent que "ce qui est vraiment $\mathrm{p}$ » et le second prédicat constituent sémantiquement une extension l'un de l'autre et entrent dans une relation de repérage qui fonctionne dans les deux sens, ce qui nous fait dire que l'objet de manque est à la fois un point de départ et un point d'arrivée notionnel dans la prédication en want. Il nous semble qu'une représentation topologique rend bien compte de ces phénomènes de prolongement et d'assimilation réciproques.

Ayant posé les grandes lignes de notre analyse à travers la problématique culiolienne de la gestion de l'altérité, nous continuons à défendre la pertinence du modèle topologique à partir de l'analyse critique d'une autre hypothèse qui, au contraire des précédentes, fonde l'émergence du désir sur l'effacement d'un prédicat non-télique.

\section{L'hypothèse d'un prédicat non-télique sous-jacent}

88 L'hypothèse de J. McCawley (1974) que nous avons présentée ci-dessus inclut en réalité des exemples où ce n'est pas tant have a $N$ qui est effacé que have $G N$, construction qui prend souvent une interprétation «moins dynamique » et dans laquelle, have ne fait plus office d'hyperonyme de verbe télique :

(50) Bill wants your apartment until June.

89 Il s'agit de dire que dans ce type d'énoncé le circonstanciel de temps, ne spécifie pas les conditions de validation de la relation prédicative Bill / want your apartment. Pour trouver un support à ce complément, McCawley suppose une proposition enchâssée contenant une relation comme Bill / have your apartment. Until June, spécifie temporellement, non pas le souhait de Bill en tant que tel, mais plutôt la période dont Bill entend jouir de l'appartement. Le prédicat envisagé n'est plus de type accomplissement. 


\section{l'effacement d'un verbe interpolé have n'est pour lui valable que lorsque le have mis en jeu} a un sémantisme plutôt « statif » et non pas de type « dynamique », comme consume.

91 A partir de (50) où le have impliqué se gloserait peut-être plus volontiers par enjoy, il semblerait plus exact de dire qu'un quantifieur comme until June s'accrocherait à un procès de type "activité », en tout cas duratif et non-télique. C'est cette seconde relation interpolée qui fonderait alors l'expression du désir en permettant d'envisager sémantiquement le dépassement du manque. Il n'est plus question de gloser le désir par une volonté de faire mais par la simple possibilité pour le sujet d'être « mis en présence » avec le référent du GN dans le virtuel. On a alors l'idée d'une palliation du manque « par l' avoir », au sens de « disposer de », ou « l'être avec ». C'est ce qui paraît effectivement être en jeu dans un énoncé comme (51), et dans les suivants :

(51) I want my MTV. (Publicité pour MTV)

(52) Nicole was a little girl again. She was very frightened. I want my mother, Nicole thought. (Arthur C. Clarke, Rama revisited)

(53) This is so serious a breach of your store's customer care that I want the manager, not just a retail rep! (Google)

(54) You want comedy ? Talk with me. (Publicité pour Channel 4)

Il s'agit d'exprimer un désir qui ne sera comblé que lorsque le référent des sujets I ou you sera confronté au référent des divers GN impliqués : my MTV, my mother, the manager, comedy, etc... Il n'y a pas prise en compte des moyens qui permettent d'atteindre l'objet de manque visé et la relation non-télique postulée ne permet pas de déterminer le GN à travers le second procès. L'objet est bien délimité référentiellement par ailleurs et jouit d'une grande autonomie syntactico-sémantique par rapport à la seconde relation LOC2 supposée effacée car le prédicat non-télique présuppose l'existence du référent du GN plutôt qu'il ne la pose.

Il nous semble que la question de la portée de la négation va contre l'idée de la présence d'une relation sous-jacente même dans ce cas :

(55) A : Do you want my skateboard?

B : I don't want a skateboard, I want a wedding dress. (entendu dans la série Shameless)

Le locuteur $A$ est un petit garçon naif qui cherche à séduire une demoiselle d'honneur partie chercher une robe. Dans l'énoncé prononcé par $\mathrm{B}$, la négation ne porte pas sur une deuxième relation de type have "statif » elle-même et "I don't want a skateboard » n'est pas exactement synonyme de "I don't want to have a skateboard ». La petite fille précise que ce qui peut la combler, c'est une robe, pas un skateboard: elle signale que le paradigme envisagé pour le référent du GN de want $G N$, n'est pas le bon. Il ne s'agit pas de nier plus ou moins directement une relation seconde qui coderait la palliation du manque par « un avoir » mais de nier le désir spécifique lui-même. Ce désir est particularisé par ce qui en constitue l'objet, si bien que nier ce désir, c'est nier son objet et vice versa, ce qui a à voir avec le fait que want est un " prédicat transitif intensionnel ».

Nous dirons donc que la prise en compte notionnelle du dépassement du manque ne nécessite pas de second prédicat effacé de quelque type que ce soit. Il est pourtant là encore possible qu'un prédicat de type have "statif ", enjoy ou be with soit reconstruit à partir de want GN dans certains contextes, ce qui témoigne d'une autre façon de donner éventuellement corps à l'altérité implicite pensée notionnellement à partir du référent du GN. Ce prédicat reconstruit n'est pourtant pas nécessaire étant donné son statut d'objet 
visé. Want GN permet justement à l'énonciateur de se placer a priori en deçà de la problématique de l'explicitation de la palliation du manque par une seconde relation.

\section{Centrage sur l'objet de manque} relations paraphrastiques que peuvent entretenir want GN et want to $V G N$. Il nous a semblé que les réécritures proposées par certains linguistes supposent un maintien de la 
notion de manque dans son programme de sens contemporain, bien que cela ne soit pas revendiqué. La conception culiolienne du domaine notionnel permet de rendre compte des gloses proposées en posant la problématique de l'altérité et de la frontière, ce qui autorise quelques prédictions sémantiques sur d'autres constructions où ce verbe apparait. L'étude des relations paraphrastiques sous cet angle pousse alors à poser que le référent du GN de la première construction n'est a priori pas distingué notionnellement d'une éventuelle seconde relation pensable à partir de lui, même si une telle relation est reconstructible. S'il y a reconstruction d'un second prédicat à partir de ce qui correspond avant tout à un objet de manque, celui-ci est fortement distingué notionnellement d'une seconde subjectivité qui sert d'objet à la volonté du sujet d'énoncé. Il est aussi faiblement distingué du référent $d u$ GN avec lequel il entretient une relation de repérage. La deuxième relation reconstructible sous certaines conditions est alors à comprendre comme l'explicitation de l'accessibilité de l'objet de manque, accessibilité qui existe par ailleurs car want permet de viser directement le référent du GN. On voit alors ce qui différencie cette conception des autres points de vue évoqués ici : prendre en compte la possibilité de concevoir ou d' "habiller» la seconde relation LOC2 de différentes manières et la possibilité de ne pas la construire.

\section{BIBLIOGRAPHIE}

Aarts, J. et F. Aarts. 1995. « Find and Want, a corpus-based study in verb complementations » in The Verb in Contemporary English - Theory and description. B. Aarts et C.F. Meyer, éds. Cambridge : Cambridge University Press : 159-182

Adamczewski, H. et C. Delmas. 1998. Grammaire Linguistique de l'Anglais. Paris : Armand Colin. 2 ème edition

Beck, S. et K. Johnson. 2004. « Double objects again » in Linguistic Enquiry 35 : 97-124

Birks, I. 2002. «L'acquisition de HAVE en L1 » in Construire et reconstruire en linguistique anglaise. Syntaxe et sémantique. C. Delmas et L. Roux, éds. Saint-Etienne : Publications de l'Université de Saint-Etienne : 285-297

Boisson, C. 1987. « Anglais 'have', français 'avoir' et l'empathie » in La Transitivité. CIEREC Travaux LII. Saint-Etienne : Publications de l'Université de Saint-Etienne, 155-180

Chuquet, J. 1986. « TO et l'infinitif anglais » in Cahiers de recherche en grammaire anglaise, T. 3. Gap : Ophrys

Cotte, P. 1998. « Have n'est pas un verbe d'action : L'hypothèse de la réélaboration » in $L a$ transitivité. A. Rousseau éd. Lille : Presses Universitaires du Septentrion : 415-439

Culioli, A. 1990. Pour une linguistique de l'énonciation. Opérations et représentations, T. 1, Paris : Ophrys Dixon, R. 1991. A Semantic Approach to English Grammar. Oxford : Oxford University Press

Evans, V. et M. Green. 2006. Cognitive Linguistics : An Introduction. Edinburgh : Edinburgh University Press 
Fodor, J. et E. Lepore. 1998. « The Emptiness of the lexicon : Reflections on James Pustejovsky's The Generative Lexicon » in Linguistic Inquiry 29 : 269-288

Franckel, J.-J.. et D. Lebaud. 1990. Les figures du sujet. A propos des verbes de perception, sentiment, connaissance. Gap : Ophrys

Gilbert, E. 1993. «La théorie des operations énonciatives d'Antoine Culioli » in Les théories de la grammaire anglaise en France. Paris : Hachette

Gilbert, E. 2001. «Vers une analyse unitaire des modalités » in Cahiers de recherche en grammaire anglaise, T. 8. Modalité et Opérations Enonciatives. Gap : Ophrys

Groussier, M.-L. et Rivière, C. 1996. Les mots de la linguistique. Lexique de linguistique énonciative. Gap / Paris : Ophrys

Guillemin-Flescher, J. 1999. « Verbes atéliques et construction d'occurrences » in Les Opérations de détermination : quantification/qualification. A. Deschamps, J. Guillemin-Flescher, éds. Gap : Ophrys, 251-268

Harley, H. 2004. « Wanting, Having, and Getting : A Note on Fodor and Lepore 1998 » in Linguistic Inquiry $35: 255-267$

Harves, S. 2008. « Intensional Transitives and Silent HAVE : Distinguishing between want and need . » in Proceedings of the 27th West Coast Conference on Formal Linguistics

Jespersen, O. 1992. The philosophy of grammar. Chicago : University of Chicago Press

Khalifa, J. C. 1999. La syntaxe anglaise aux concours, Théorie et pratique de l'énoncé complexe. Paris : Armand Colin

Krug, M. 2000. Emerging English Modals : a corpus-based study of grammaticalization. Berlin et New York : Mouton de Gruyter

Kuno, S. 1987. Functional syntax, Anaphora, Discourse and Empathy. Chicago : The University of Chicago Press

Lalande, A. 2006. Vocabulaire technique et critique de la philosophie. Paris : PUF. $2^{\text {ème }}$ edition Larreya, P. 1989. «Sur la relation be / have », in L'Anaphore, Domaine anglais. Saint-Étienne : CIEREC : $59-77$

Lyons, J. 1995. Linguistic semantics : An introduction. Cambridge : Cambridge University Press

Marušič, F. et R. Žaucer. 2006. « On the complement of the intensional transitive 'want' » in Stony Brook Occasional Papers in Linguistics 1. T. Kawamura, Y. Suh, et R. Larson, éds. Stony Brook : 128-151

McCawley, J. 1974. « On identifying the remains of deceased clauses » in Language Research 9: 73-85

Merle, J.-M. 2003, «Want », in Linguistique comparée et traduction, Tome 6. Gap : Ophrys : 151-181

Muller, P. 2011. Elements pour une caractérisation abstraite du verbe anglais Want. Thèse Paris 3

Polge. 2006. « La subjectivisation de want et les niveaux notionnel, predicative et énonciatif » in Constructions verbales et production de sens. D. Lebaud, C. Paulin et K. Ploog, éds. Besançon : Presses universitaires de Franche-Comté : 349-359

Polge. 2007. Etude du prédicat Want et de ses emplois en anglais contemporain. Thèse Paris 7

Polge. 2008. «Want: du manque au désir » in Du notionnel à l'énonciatif et retour, Cahiers de Recherche en linguistique anglaise. M.-L. Groussier et C. Rivière, éds. Paris : Ophrys : 107-125 
Pustejovsky, J. 1995. The Generative Lexicon. Cambridge, Mass. : MIT Press

Quine, W. V. O. 1960. Word and Object. Cambridge, Mass. : MIT Press

Quirk, R. et al. 1985. A Comprehensive Grammar of English. New York : Longman

Ross, J.R. 1976. « To have 'have' and to not have 'have'« in Linguistic and literary studies in honor of Archibald A. Hill. 1. M. A. Jazayery, E. Polomé, et W. Winter éds. The Hague : Mouton ; 263-270

Sugisaki, K. 2009. « On Children's NEEDs » in Proceedings of the Seventh GLOW in Asia. R. Mohanty et M. Menon éds. Hyderabad : EFL University Press : 225-236

Traugott, E. 1989. « On the rise of epistemic meanings in English : An example of subjectification in semantic change ", in Language $57: 33-65$

Vendler, Z. 1967. Linguistics in Philosophy. Ithaca, NY : Cornell University Press

Wechsler, S. 2005. «Wanting, Getting, and Enjoying it ». Handout from talk presented at the University of Berkley. https://webspace.utexas.edu/wechsler/BerkeleyHAVE4.handout.pdf -

Wierzbicka, A. 1996. Semantics : Primes and Universals. Oxford : Oxford University Press

\section{NOTES}

1. Il s'agit dans les théories énonciatives d'une opération de repérage dissymétrique entre deux termes irréductibles dont l'un prend le statut de repère et l'autre celui de repéré. (M.-L. Groussier et C. Rivière (1996)).

2. Nous faisons référence au QLT Culiolien lié au repère $S$ des coordonnées énonciatives et qui a à voir avec le discernement de ce qu'est la chose - la construction de propriétés, de ce qui la fait exister en tant que chose - par opposition à QNT, lié au repère $\mathrm{T}$, et qui a à voir avec la division de l'espace-temps, et la construction d'occurrences situationnelles, voir par exemple E. Gilbert (2001: 24-25). La mise en relation argumentale permise par want et la réanalyse pragmatique sont ce qui permet de donner le référent de $\mathrm{S}$ en sujet désirant $\mathrm{p}$ plutôt que simplement manquant de $\mathrm{p}$.

3. Ce qui se fait suivant un postulat très simple : « if a noun phrase can denote a desired entity, so too can an infinitive " (M. Krug (2000: 144)).

4. K. Sugisaki (2009) va dans le même sens en soulignant le fait que les enfants acquièrent want le plus souvent avant have. Son étude montre aussi que have est acquis avant need (parfois en même temps mais jamais après).

5. Faire du complément de want une proposition réduite («small clause ») peut faire pencher pour la seconde analyse (voir (18)) mais accorder le statut de construction de « plein droit » à (15) peut faire préférer l'analyse plus descriptive de in en préposition. R. Quirk et al. (1985 : 732) en font un élément au fonctionnement adverbial, ce qui n'est pas contradictoire avec les deux hypothèses.

6. Nous avons trouvé 3 occurrences de cette séquence sur Google mais il s'agit de cas marginaux où want est rapproché - pour être distingué - de need à la voix passive : "As a teacher of high schoolers, I can say that there are very few occasions where an IPad is needed, but 100 times where an IPad is wanted...by other students. »

7. Il s'agit d'examiner la problématique du dépassement du manque à travers celle de la construction d'une altérité à partir de $\mathrm{p}$, au sens culiolien du terme. Ceci permet d'envisager l'accessibilité de $\mathrm{p}$ en relation avec une seconde subjectivité qui permet d'agir comme "relais » du sujet de want dans la complémentation. 
8. Les sections suivantes vont envisager différentes façons de concevoir la relation LOC2 et son explicitation dans les gloses mais nous défendrons aussi la possibilité de sa non-construction, l'objet de manque sera alors visé directement.

9. C'est-à-dire "ce qui tend à une fin » ou encore une " puissance d'action dirigée en un sens défini mais qui ne s'actualise pas, ou du moins pas entièrement » (Lalande (2006)).

10. C'est pourquoi J.-M. Merle (2003), reprenant la terminologie que J.-J. Frankel et D. Lebaud (1990) avaient proposée pour désirer, appelle le S1 de want «sujet d'indexation " alors que S2 correspond au « sujet d'effectuation » dans la mesure où le premier est celui à qui est rapportée la relation nécessaire alors que le second est l'agent de la relation qui va lui servir de relais dans la partie de la construction associable avec la palliation du manque.

11. On peut ainsi parler de «structure manipulative » pour la deuxième construction, comme le fait J.-C. Khalifa (1999).

12. "The windowing pattern involves the explicit mention of some parts of a scene (windowing), while other parts may be omitted (gapping). » (V. Evans et M. Green (2006:527)).

13. Nous considérons ce modèle connu et signalons simplement que l'on en trouve une présentation très claire chez E. Gilbert (1993 : 70-73) par exemple.

14. Nous sommes responsable de cette interprétation car les travaux que nous citons n'expliquent pas de manière notionnelle pourquoi elles défendent ces réécritures systématiques. Elles ne traitent pas clairement de la notion du manque et de son dépassement mais il nous semble qu'elles reposent sémantiquement sur le non-dit que nous venons d'évoquer.

15. Ainsi, pour J. Guillemin-Flescher (1999 : 251) les procès téliques sont à associer à des propriétés qui impliquent un dernier point.

16. Ainsi, il nous semble que «I want $a$ dog " pourrait à la rigueur s'interpréter comme «I want to buy a $\operatorname{dog}$ ", qui réfère à un accomplissement, mais pas comme «I want to walk a dog» qui renverrait à une activité.

17. La télicité du prédicat reconstruit suppose donc un lien privilégié entre la notion à laquelle renvoie le GN en position d'objet et celle évoquée par le prédicat verbal transitif, lien qui est tout sauf accidentel, et l'ensemble forme alors un domaine notionnel plus vaste : « [...] chaque fois qu'un énonciateur effectue un choix entre notions, puis construit des relations non-accidentelles entre ces notions (autrement dit construit des relations prédicatives entre notions), il délimite des domaines différents et par là même les complémentaires des choix effectués. La relation < ( ) smoke, meat > est en quelque sorte un sous-domaine de / ( ) ( ) smoke / et fonctionne à son tour comme une notion : son choix est le choix de tout ce qui illustre cette relation (on peut parler de classe d'occurrences) à l'exclusion de tout ce qui ne l'illustre pas. » (J. Chuquet (1986: 95))

18. Voir aussi 15 ci-dessous.

19. Ce que notait déjà A. Wierzbicka (1996: 119).

20. Possibilité qui avait été envisagée par J. Ross (1976 : 264), bien qu'il ait été lui-même partisan de la " have deletion» : " [...] the alternative to a syntactic rule of HAVE DELETION would be a semantic rule which would convert the single-clause syntactic structure [...] into the semantically necessary bipropositional structure [...]. That is, there can be no choice on grounds of simplicity between a syntactic rule of HAVE DELETION and an interpretive semantic rule of proposition building, for the operations are essentially inverses of one another. "

21. F. Maružič et R. Žaucer (2006) montrent que l'hypothèse de Harley s'appuie sur une extension des travaux de S. Beck et K. Johnson (2004) qui expliquent le "sens de transfert» associé généralement à la syntaxe ditransitive par l'existence d'une proposition réduite en have sousjacente. 


\section{RÉSUMÉS}

Après avoir rappelé quelques éléments diachroniques quant à l'émergence des notions de désir et de volonté pour want, verbe associé primitivement au manque, le présent article se fixe comme objectif d'étudier la sémantique contemporaine de la construction want GN. Il sera proposé que la notion de manque doit être intégrée à son programme de sens moderne, ce qui constitue l'un des non-dits des hypothèses qui proposent pour cette construction des réécritures systématiques à l'aide d'un élément effacé (qui va du verbe à la préposition abstraite) et dont l'objectif semble être de rendre compte du dépassement du manque par une seconde relation. L'analyse critique de ces hypothèses pousse à remettre en question la systématicité des effacements postulés. Il sera proposé, dans une approche énonciativiste, que l'opération de visée, d'une part, et le modèle topologique du domaine notionnel d'autre part, permettent de rendre compte du dépassement du manque et d'expliquer l'émergence des notions connexes que sont le désir et la volonté. La problématique de la gestion d'une altérité cognitive posée à travers ce modèle est en outre ce qui permet d'effectuer des prédictions sur le sens de certaines constructions en want non traitées dans ces approches.

After reintroducing some diachronic elements about the development of the notions of desire and volition for want - a verb originally associated with an idea of deficit or lack - this paper aims to study the semantics of the want NP construction. It is proposed that the notion of deficit should be integrated into its modern meaning, a point which is only tacitly acknowledged in some hypotheses arguing that this construction can be systematically rephrased with the help of a deleted element - ranging from a verb to an abstract preposition. These approaches are understood as trying to account for the fact that the notions want NP expresses today are semantically always situated beyond that of deficit, a state of affairs which is achieved through a covert embedded relation. These views are first critically assessed so that their systematicity is questioned. It is then proposed, in an enunciative approach, that the concept of prospective validation and Culioli's topological representation of the notional domain are relevant to account for the construction of the notions of desire and volition from that of deficit. This claim raises the issue of the management of cognitive otherness, which is central in predicting the meaning of some want-constructions that are not addressed in those hypotheses.

\section{INDEX}

Keywords : want, gloss, notional domain, otherness, nexus, prospective validation, deficit, polysemy, invariance

Mots-clés : glose, have, domaine notionnel, altérité, visée, manque, polysémie

\section{AUTEUR}

\section{PHILIPPE MULLER}

Université Bordeaux 3, Michel de Montaigne - équipe CLIMAS (EA 4196) 